Z. Klin. Chem. Klin. Biochem.

12. Jg. 1974, S. $128-133$

\title{
Radioimmunologische Bestimmung von Aldosteron im Plasma ${ }^{1}$ )
}

Von F. Klumpp, R. Rössler und D. Klaus

Medizinische Poliklinik der Universität Marburg und Medizinische Poliklinik der Universität Tübingen

(Eingegangen am 10. August 1973/2. Januar 1974)

Herrn Professor Dr. med. F. Heni zum 65. Geburtstag

\begin{abstract}
Es wird eine modifizierte radio immunologische Methode für die Bestimmung der Aldosteronkonzentration beschrieben, für die $2 \mathrm{ml}$ Plasma erforderlich sind. Die Aldosteron-Antikörper wurden vom Kaninchen gewonnen, die Extraktion des Aldosterons aus dem Plasma erfolgte mit Dichlormethan und anschließender Chromatographie in Benzol-Methanol-Wasser. Dabei trat ein Aldosteronverlust von im Durchschnitt 50\% auf, der in jedem Ansatz durch Zusatz einer bekannten Menge von [1,2- $\left.{ }^{3} \mathrm{H}\right]$ Aldosteron genau berechnet werden konnte. Der radioimmunologische Ansatz erfolgte mit einem 1:400 verdünntem Antiserum. Die Trennung von freiem und an Antikörper gebundenem $\left[1,2-{ }^{3} \mathrm{H}\right]$ Aldosteron wurde mit einer Kohle-Dextran-Suspension durchgeführt. Die Genauigkeit der Methode und die Wiederfindungsrate von zugesetztem Aldosteron wurden getrennt in 2 Laboratorien geprüft. Untersuchungen über die Kreuzreaktion der Antikörper mit anderen Steroiden zeigen, daßB durch das angewandte Verfahren eine ausreichende Isolierung des Aldosterons stattfindet. Bei 35 Gesunden erhielten wir eine Plasma-Aldosteron-Konzentration von $154 \pm 57 \mathrm{ng} / 1$. Bei 3 Patienten mit unbehandeltem Morbus Addison war die Plasma-Aldosteron-Konzentration auf Werte unter $10 \mathrm{ng} / \mathrm{l}$ vermindert, bei 2 Patienten mit Aldosteron-sezernierenden Nebennierenrindenadenomen auf das 4-6fache erhöht.
\end{abstract}

\section{Radioimmunassay of aldosterone in plasma}

A radioimmunological method for the estimation of the plasma-aldosterone level in man, using $2 \mathrm{ml}$ of plasma, is described. The aldosterone antibodies were obtained from rabbits. The aldosterone is extracted from plasma by dichloromethane. Subsequent chromatography is performed in benzene-methanol-water. The mean loss of aldosterone is $50 \%$. The loss during the extraction procedure was exactly calculated by adding a known amount of $\left[1,2-{ }^{3} \mathrm{H}\right]$ Aldosterone to every sample before extraction. The radioimmunassay was performed with an antiserum diluted 1:400. The separation of free and bound aldosterone was accomplished by a charcoal-dextran suspension.

The accuracy of the method, tested in 2 different laboratories lies below $18 \%$. Tests on cross reaction of antibody with other steroids show that the method provides a sufficient separation of aldosterone. In 35 healthy persons we found an aldosterone plasma level of $154 \pm 57 \mathrm{ng} / \mathrm{l}$. In 3 untreated patients suffering from adrenal insufficiency the aldosterone plasma level was less than $10 \mathrm{ng} / 1$ and in 2 patients with aldosterone-producing adenomas of the adrenal gland it was 4-6 times higher than in normal persons.

Radioimmunologische Verfahren haben sich in der klinischen Chemie für die Bestimmung von Hormonen und Substanzen, die in Körperflüssigkeiten in kleinsten Konzentrationen vorliegen, einen festen Platz erobert. Auch für den Nachweis von Aldosteron im Plasma wurden radioimmunologische Verfahren $(1,2)$ entwickelt, deren Hauptschwierigkeit weniger in der radioimmunologischen Bestimmung von Aldosteron selbst, sondern in der Extraktion des Hormons aus dem Plasma und der Erfassung des dabei auftretenden Verlustes liegt. Ein Nachteil der von Bayard et al (1) angegebenen Methode besteht in der großen Plasmaausgangsmenge von $5 \mathrm{ml}$, die für die Bestimmung erforderlich ist und in der Schwierigkeit, den Verlust von Aldosteron in der Einzelprobe genau zu erfassen. Der Nachteil der Methode von Mayes (2) besteht beim immunologischen Ansatz in der ungenügenden Trennung von freiem und gebundenem Aldosteron durch Ammoniumsulfat. Wir haben daher eine Methode entwickelt, die die Vorteile beider angegebener Verfahren vereinigt und ihre Nachteile vermeidet.

\section{Methodik}

Herstellung der Antikörper nach Bayard et al (1) Acetylierung des Aldosteron-21-monoacetat (3)

$10 \mathrm{mg}$ Aldosteron-21-monoacetat ${ }^{2}$ ) wurden bei $37^{\circ} \mathrm{C}$ für 24 Stunden in einem Vakuumexsiccator, der $\mathrm{CaCO}_{3}$-Granula enthält, mit $5 \mu 1$ Essigsäureanhydrid (Merck Nr. 42) und $5 \mu l$ Pyridin (Merck Nr. 7460) inkubiert. Dabei findet eine Acetylierung statt und es entsteht Aldosteron-18-21-diacetat. Das Gemisch wird dann in $0,5 \mathrm{ml}$ bidest. Wasser gelöst und mit 8 Vol-Teilen Dichlormethan und 8 Vol-Teilen bidest. Wasser gereinigt.

Nach Eindampfen des Dichlormethans bleibt als Rückstand gereinigtes Aldosteron-1 8-21-diacetat zurück.

\section{Herstellung eines Aldosteron-Albumin-Komplexes}

Das aus 10.mg Aldosteron-monoacetat gewonnene Aldosterondiacetat wurde mit $10 \mathrm{mg}$ Natriumacetat (Merck Nr. 6268) und $5 \mathrm{mg}$ Carboxy-methoxylamin-hemihydrochlorid (CalBiochem) versetzt. Das Gemisch wurde mit $5 \mathrm{ml}$ Methanol für 18 Stunden bei Raumtemperatur inkubiert. Das dabei entstandene Aldosteron-3-Carboxymethoxim-18-21-diacetat

\footnotetext{
1) Mit Unterstützung der Deutschen Forschungsgemeinschaft

2) Der Firma Ciba. A.G., Grenzach/Baden danken wir für die Uberlassung von Aldosteron und Aldosteron-21-monoacetat.
} 
wurde in $20 \mathrm{ml}$ bidest. Wasser gelöst und mit $200 \mathrm{ml}$ Essigsäureaethylester (Merck Nr. 9623) extrahiert. Der Extrakt wurde im Rotationsverdampfer eingedampft. Zum mit $0,5 \mathrm{ml}$ $\mathrm{H}_{2} \mathrm{O}$ aufgelösten Rückstand wurden $100 \mathrm{mg}$ Kaninchen- $\mathrm{Al}-$ bumin und $200 \mathrm{mg} 1-\mathrm{A}$ thyl-3-( $\beta$-dimethylaminopropyl)-carbodiimidhydrochlorid, gelöst in $0,25 \mathrm{ml} \mathrm{H}_{2} \mathrm{O}$, hinzugefigt. Diese Carbodiimidierung ist fuir die Koppelung des Aldosterons an das Protein notwendig $(4,5)$. Das Gemisch wurde dann mit $5 \mathrm{ml}$ bidest. Wasser verdünnt. Anschließend erfolgte eine 48 stündige Dialyse gegen $201 \mathrm{H}_{2} \mathrm{O}$ bei $4^{\circ} \mathrm{C}$.

\section{Immunisierung}

Es wurde eine Emulsion des Protein-Steroid-Komplexes (110 mg) mit $5 \mathrm{ml}$ Freund'schem Adjuvans hergestellt. Von dieser Emulsion injizierten wir jedem von 5 Kaninchen je $1 \mathrm{ml}$ subcutan in den Nacken. Es erfolgten 6 Injektionen in 4 bis 5 wöchigen Abständen.

\section{Antikörpertestung}

Die Testung der Kaninchenseren auf Antikörper gegen Aldosteron erfolgte ab der 4 . Injektion. Nur bei einem von $5 \mathrm{Ka}$ ninchen wurden Antikörper erhalten, die erstmals 7 Tage nach der 4. Injektion festgestellt werden konnten und nach weiteren 2 Injektionen den höchsten Titer erreichten. Das Serum dieses Tieres wurde in $0,1 \mathrm{ml}$ Portionen bei $-22^{\circ} \mathrm{C}$ aufbewahrt; der Antikörpertiter hat sich seit 18 Monaten nicht geändert.

Zur Prüfung der Kaninchenseren auf Antikörper wurden zunehmende Serumverdünnungen mit verschiedenen $\left[1,2^{3} \mathrm{H}\right]$ Aldosteron-Mengen inkubiert $\left(\left[1,2^{3} \mathrm{H}\right]\right.$ Aldosteron, spezifische Aktivität von 50-52 Ci/mMol, Fa. Amersham, England). Die zugefügte $\left[1,2^{3} \mathrm{H}\right]$ Aldosteronmenge, verdünnt in ly sozymhaltigem $(0,1 \%) 0,1 \mathrm{~mol} / 1$ Tris-Acetatpuffer, $\mathrm{pH} 7,4$, betrug $500,1000,1500,2000$ und $4000 \mathrm{Imp}$. $/ \mathrm{min}$. Wir verwendeten Serumverdünnungen von 1:100, 1:400, 1:800 und 1:1600. Im Testansatz wurden $0,1 \mathrm{ml}$ Serumverdünnung und $0,1 \mathrm{ml}$ $\left[1,2-{ }^{3} \mathrm{H}\right]$ Aldosteron in einem Gesamtvolumen von $0,5 \mathrm{ml}$ (aufgefullt mit Tris-Acetatpuffer) für eine halbe Stunde bei Raumtemperatur und 2 Stunden bei $4^{\circ} \mathrm{C}$ inkubiert. Nach der Inkubation wurde eine Trennung mit $0,5 \mathrm{ml}$ Kohle-DextranSuspension (400 mg Aktivkohle und $40 \mathrm{mg}$ Dextran 70 in $100 \mathrm{ml}$ Tris-Acetatpuffer $\mathrm{pH} 7,4$ ) durćhgeführt. Das nicht an Antikörper gebundene $\left[1,2-{ }^{3} \mathrm{H}\right]$ Aldosteron wird hierbei an die Kohle adsorbiert. Nach 10 minütiger Kontaktzeit bei $4^{\circ} \mathrm{C}$ werden die Proben für 20 Minuten bei $4^{\circ} \mathrm{C}$ und bei $3000 \mathrm{~g}$ zentrifugiert. Der Überstand, d. h. die antikörpergebundene Fraktion des $\left[1,2{ }^{3} \mathrm{H}\right]$ Aldosterons wurde im Flüssigkeitsszintillationszähler (Fa. Packard) je 10 Minuten lang gezählt. Als Szintillationsflüssigkeit (10 $\mathrm{ml}$ je Probe) verwendeten wir ànfangs Bray'sche Lösung, später Insta-Gel (Fa. Packard). Die stärkste Bindung erhielten wir bei einer Serumverdünnung von 1: 400 und bei einer $\left[1,2-{ }^{3} \mathrm{H}\right]$ Aldosteronmenge mit einer Impulsrate von 1200 bis $1700 \mathrm{Imp}$./min, die etwa 8-10 pg markiertem Aldosteron entspricht. Die Bindung der zugefügten Aktivität betrug bei der genannten Verdünnung und Aktivität $50-70 \%$. Bei stärkerer Verdünnung des Antiserums und bei höherer Aktivität des zugefügten $\left[1,2-{ }^{3} \mathrm{H}\right]$ Aldosterons war die maximale Bindung vermindert.

\section{Extraktion und Chromatographie des Aldosterons aus} dem Plasma

$\mathrm{Zu} 2 \mathrm{ml}$ Heparinplasma wurden etwa 15000-17000 Imp./min $\left[1,2^{3} \mathrm{H}\right]$ Aldosteron (entspricht etwa $100 \mathrm{pg}$ Aldosteron) ge löst in Äthanol und $50 \mu \mathrm{l} \mathrm{NaOH}$ hinzugefuigt (Tab. 1). Diese Lösung wurde zweimal mit je $40 \mathrm{ml}$ Dichlormethan extrahiert. Um das Aldosteron möglichst vollständig zu extrahieren, wird zweimal je 10 Minuten geschüttelt. Der Dichlormethan-Extrakt wurde anschließend einmal mit $70 \mathrm{ml} 0,1 \mathrm{~mol} / 1$ Essigsäure und zweimal mit $70 \mathrm{ml}$ bidest. Wasser gewaschen. Der DichlormethanExtrakt wurde dann im Becherglas im Abzug eingedampft. Der Ruickstand wurde in $1 \mathrm{ml}$ Methanol aufgelöst und auf Chromatographiepapier aufgetragen (Nr. $2043 \mathrm{a} \mathrm{mgl,} \mathrm{Fa.} \mathrm{Carl} \mathrm{Schleicher}$ und Schüll). Vor dem Auftragen des Methanolextraktes wurde das Papier mit Methanol gewaschen. Die Chromatographie wurde durchgefuihrt im Bush-System $B_{5}$ (Benzol:Methanol: Wasser Volumina $=20 \mathrm{ml}+10 \mathrm{ml}+10 \mathrm{ml}$ ). Die Dauer der
Tab. 1. Extraktion und Isolierung des Aldosterons aus dem Plasma

\section{$2 \mathrm{ml}$ Plasma}

$+\left\{\begin{array}{l}50 \mu 1 \mathrm{NaOH} \\ 15000 \mathrm{Imp} . / \mathrm{min}\left[1,2^{-3} \mathrm{H}\right] \text { Aldosteron }\end{array}\right.$

Extraktion mit $2 \mathrm{mal} 40 \mathrm{ml}$ Dichlormethan

Waschen mit $0,6 \mathrm{~mol} / 1$ Essigsäure $(80 \mathrm{ml})$

Waschen mit $2 \mathrm{mal} 80 \mathrm{ml}$ bidest. Wasser

Eindampfen des Dichlormethanextraktes

\author{
Papierchromatographie im Bush-System \\ Extraktion des Aldosterons aus dem Papier mit Methanol \\ Eindampfen des Methanolextraktes
}

absteigenden Chromatographie betrug 6-8 Stunden bei Raumtemperatur $(6,7,8)$. Der $R_{F}$-Wert für Aldosteron betrug in diesem System 0,35-0,37. Das zum Vergleich mitchromatographierte Cortisol hatte einen $R_{F}$-Wert von $0,22-0,25$, Cortison 0,42-0,44. Das radioaktive Aldosteron wird nach der Chromatographie im Papierstreifenabtaster (Scanner der Fa. Packard) lokalisiert. (Bei gleichzeitiger Chromatographie von $\left[1,2-{ }^{3} \mathrm{H}\right]$ Aldosteron, Cortisol und Cortison kann gezeigt werden, daß die Aldosteron-Bande zwischen der Cortisol- und Cortison-Bande liegt, die im UV-Licht nach Besprühung des $\mathrm{Pa}$ piers mit 15 proz. Phosphorsäure-Spray fluoreszieren). Nach der Lokalisation von Aldosteron mit Hilfe des Scanners wurden $3 \mathrm{~cm}$ des Aldosteron-Peaks ausgeschnitten. Das Papierstückchen wurde in $10 \mathrm{ml}$ Methanol eluiert. Das Eluat wurde eingedampft, der Bodensatz wurde mit $2 \mathrm{ml} \mathrm{0,1} \mathrm{mol} / 1$ TrisAcetatpuffer (pH 7,4 mit 0,1\% Lysozym) aufgelöst. Hiermit erfolgt der radioimmunologische Ansatz: Im Verlauf der Extraktion und Chromatographie tritt ein Verlust von $50 \%$ auf, der bei der Berechnung berücksichtigt wurde.

\section{Radioimmunologischer Ansatz und Herstellung der} Standardkurve

Für den radioimmunologischen Ansatz (Tab. 2) wurden jeweils $0,4 \mathrm{ml}$ des erhaltenen Eluates verwendet (Doppelbestimmung). Dies entspricht bei einem Verlust von $50 \%$ während der Isolierung des Aldosterons ungefähr einem Zehntel der zugefügten $\left[1,2-{ }^{3} \mathrm{H}\right]$ Aldosteronmenge. Der Schwankungsbereich des Gehaltes der Plasmaansätze an markiertem Hormon liegt zwischen 980 und $1890 \mathrm{Imp}$./min mit einem Mittelwert von $1570 \pm 230 \mathrm{Imp}$. $/ \mathrm{min}\left[1,2^{3} \mathrm{H}\right]$ Aldosteron (siehe Tabelle 2a). Liegt die Ausbeute nach Chromatographie außerhalb dieses Bereichs, wird diese Probe nicht berücksichtigt. $\mathrm{Zu}$ diesen Proben wurden 0,1 ml von 1: 80 verdünntem Antiserum zugegeben. (Endverdünnung des Antiserums in Inkubationsansatz 1:400). Die Proben wurden 30 Minuten bei Raumtemperatur und 2 Stunden bei $4^{\circ} \mathrm{C}$ inkubiert. Parallel dazu wurde die gleiche Menge des Plasmaeluates $(0,4 \mathrm{ml} \mathrm{mit}$ $1500 \mathrm{Imp}$./min markiertem Aldosteron) ohne Antiserum in einem Gesamtvolumen von $1 \mathrm{ml}$ zur Gewinnung des $100 \%$ Bezugswertes angesetzt (siehe Tabelle 2). Zur Bestimmung der Recovery wurden $0,4 \mathrm{ml}$ des Plasmaeluates ohne Antiserum sofort in ein Szintillationsgefäß gegeben.

Zur Gewinnung der Standardkurve wurden bekannte Mengen von unmarkiertem Aldosteron $(12,5 \mathrm{pg}, 25,50,100,200$, $400 \mathrm{pg}$ ) mit $0,1 \mathrm{ml}$ verdünntem Antiserum und $0,1 \mathrm{ml}$ $\left[1,2{ }^{3} \mathrm{H}\right]$ Aldosteron (1500 Imp./min) in einem Gesamtvolumen von $0,5 \mathrm{ml}$ eine halbe Stunde bei Raumtemperatur und 2 Stunden bei $4^{\circ} \mathrm{C}$ inkubiert. Als $100 \%$ Bezugswert diente ein Ansatz mit $1500 \mathrm{Imp} . / \mathrm{min}\left[1,2-{ }^{3} \mathrm{H}\right]$ Aldosteron ohne Antiserum in einem Gesamtvolumen von $1 \mathrm{ml}$. Nach der Inkubation wurden zu den Proben der Standardkurve und den Plasmaproben 0,5 ml Kohle-Dextranlösung zugefügt, jedoch nicht zu denjenigen Proben, die für die Gewinnung des $100 \%$-Bezugswertes angesetzt wurden. Nach sorgfältigem Schütteln wurden die Proben 
Tab. 2. Immunologischer Ansatz zur Aldosteronbestimmung

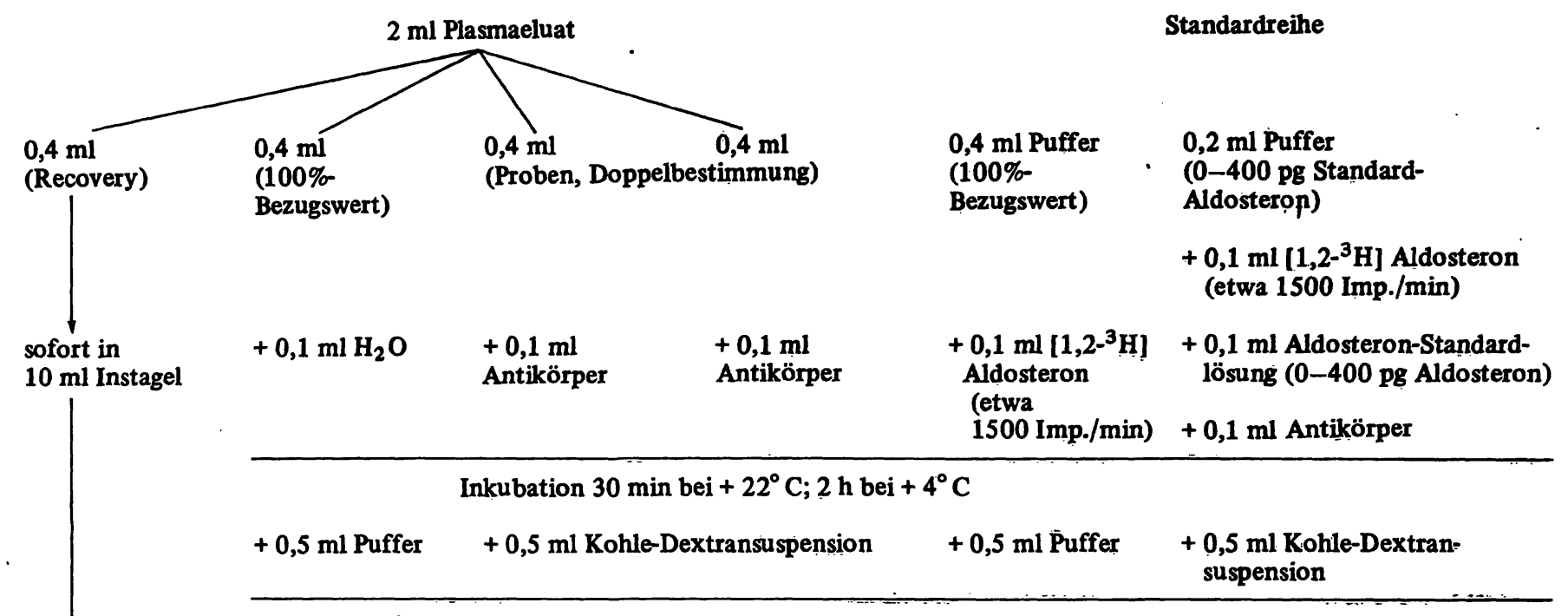

$10 \mathrm{~min}$ bei $4^{\circ} \mathrm{C}$ stehen lassen; 10 min zentrifugieren;

Überstand in $10 \mathrm{ml}$ Instagel zählen

10 Minuten bei $4{ }^{\circ} \mathrm{C}$ und $3000 \mathrm{~g}$ zentrifugiert. Die Aktivität im Überstand (an Antikörper gebundene Aktivität) und die Aktivität in den Proben für den 100\%-Bezugswert sowie in den Proben des Plasmaeluates wurden im Flüssigkeitsszintillationszähler bestimmt (Zählung: 10 Minuten). Zur Herstellung der Standardkurve wurden die bei den einzelnen Aldosteronmengen gefundenen Werte in Prozent des 100\%-Bezugswertes umgerechnet und auf Millimeterpapier aufgezeichnet.

Entsprechend wurden für jede Plasmaprobe das prozentuale Verhältnis der gebundenen Fraktion gegenüber dem zugehörigen Plasmabezugswert errechnet und an der Standardkurve die entsprechende Menge Aldosteron in pg abgelesen. Aus der am Anfang des Plasma zugefügten $\left[1,2-{ }^{3} \mathrm{H}\right]$ Aldosteronmenge (zugefügtes Aldosteron in Imp./min), der Aktivität des für den immunologischen Ansatz verwendeten Eluates (EluatAktivität in Imp./min), dem Plasmaausgangsvolumen (eingesetztes Plasma in $\mathrm{ml}$ ) und dem in der Standardkurve abgelesenen Wert A (in pg Aldosteron) läßt sich dann die Aldosteronkonzentration in ng/l Plasma umrechnen nach der folgenden Formel:

Aldosteronkonzentration (ng/l)

$=$ Aldosteron (pg) $\cdot$ zugefügtes Aldosteron (Imp. $/ \mathrm{min})$ $=\frac{\text { Eluat-Aktivität (Imp./min) } \cdot \text { eingesetztes Plasma (ml) }}{\text { El }}$

\section{Ergebnisse}

\section{Standardkurve}

Die maximale Bindung der zugefügten $\left[1,2 \cdot{ }^{3} \mathrm{H}\right]$ Aldosteron-Aktivität betrug mit unserem Antiserum bei einer Endverdünnung 1:400 50-70\%; bei Zugabe einer Aktivität von $1200-2000 \mathrm{Imp} . / \mathrm{min}\left[1,2-{ }^{3} \mathrm{H}\right]$ Aldosteron entsprechend der Aktivität der Plasmaeluate (Tab. 2a) zeigen die Standardkurven einen gleichartigen Verlauf (Abb. 1). Die Standardkurve läßt sich durch Logit-Transformation mit Auftragen der Standardkonzentration des Aldosterons in logarithmischen Maßstab in eine Gerade umwandeln (9); (siehe Abb. 2). Im Bereich von 12,5 bis $200 \mathrm{pg}$ erlaubt die Logit-Transformation eine höhere Ablesegenauigkeit.
Tab. 2a. Aktivität der Plasmaeluate $(0,4 \mathrm{ml})$ nach Chromatographie

\begin{tabular}{llll}
\hline Probe Nr. & Imp./min & Probe Nr. & Imp./min \\
\hline 1 & 1400 & 13 & 1810 \\
2 & 1890 & 14 & 1330 \\
3 & 1695 & 15 & 1620 \\
4 & 1780 & 16 & 1820 \\
5 & 1420 & 17 & 1880 \\
6 & 1460 & 18 & 1630 \\
7 & 1260 & 19 & 1450 \\
8 & 1580 & 20 & 1880 \\
9 & 1800 & 21 & 1540 \\
10 & 1315 & 22 & 1420 \\
11 & 1660 & 23 & 1540 \\
12 & 980 & 24 & 1650 \\
\hline
\end{tabular}

$\bar{x} \pm s=1570 \pm 230 \mathrm{Imp} . / \mathrm{min} ; \mathrm{n}=24$

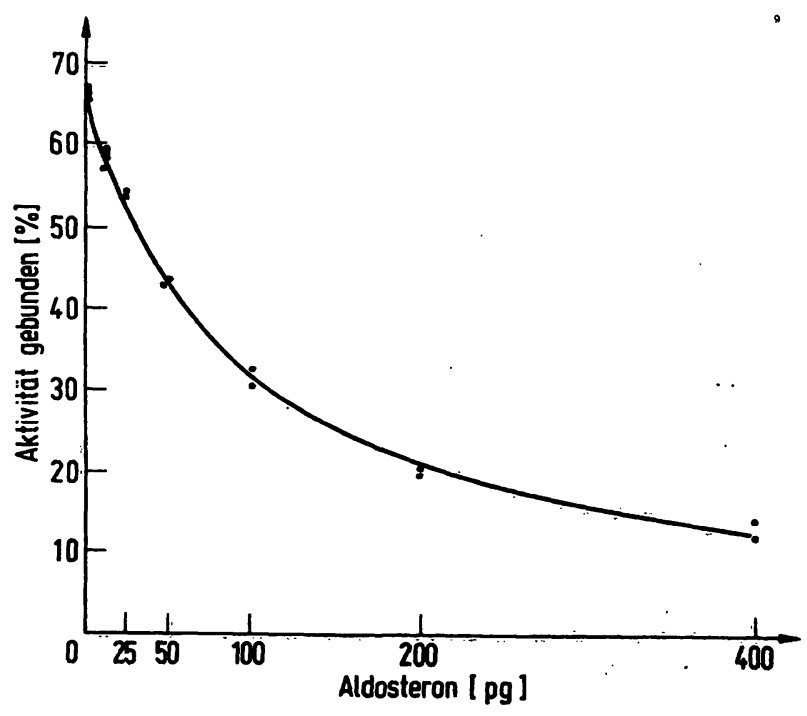

Abb. 1. Standardkurven bei einer Antiserumverdünnung von 1:400 und der zugefügten Aktivität von $1200 \mathrm{bzw}$. $2000 \mathrm{Imp}$./min $\left[1,2^{3} \mathrm{H}\right.$-] Aldosteron. 


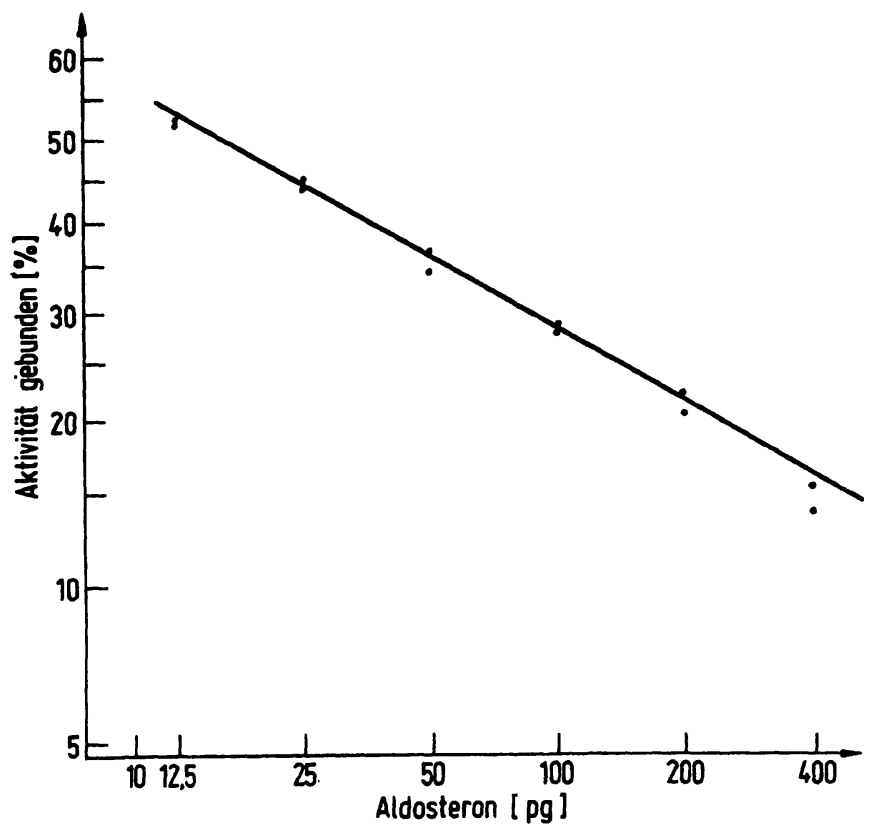

Abb. 2. Logit-Log-Transformation derselben Standardkurve

\section{Empfindlichkeit, Richtigkeit und Präzision der Methode}

Die Empfindlichkeit der Methode (Sensitivität) läßt die Bestimmung von $5 \mathrm{ng}$ Aldosteron im Plasma als niedrigster von Null unterscheidbarer Konzentration zu. Die bei jeder Serie mitgefuhrten Leerwerte liegen unter $50 \mathrm{ng} / \mathrm{l}$; der Einsatz eines reinen Papiereluats ergab 0 ng/l Aldosteron. Die Fehlerbreite des radioimmunologischen Ansatzes beträgt bei Doppelbestimmungen $\pm 11,8 \%$. Die Fehlerbreite der gesamten Methode einschließlich der Aldosteronextraktion aus dem Plasma, die mit je 6 Ansätzen von bekannten Mengen nicht markierten Aldosterons $(62,5,125,250,500$ und $1000 \mathrm{pg}$ ) bestimmt wurde, ist mit $\pm 22 \%$ etwas höher (Abb. 3).

Eine Studie über die Präzision der Methode wurde durch vergleichende Bestimmungen von Plasmaproben in unseren beiden Laboratorien durchgeführt. Es ergab sich eine gute Ubereinstimmung der Werte (Tab. 3). Die mittlere prozentuale Differenz der Einzelwerte betrug $\pm 17,7 \%(10)$.

Kreuzreaktion mit anderen Steroiden (Spezifität der Methode

Das von uns hergestellte und verwendete Antiserum weist eine Kreuzreaktion mit Corticosteron, Cortisol und Cortison auf. Die Reaktion dieser Steroide in 1000fach höherer Konzentration mit dem Antikörper zeigt Abbildung 4. Bei Annahme einer 50\%igen Bindung von Aldosteron beträgt die Kreuzreaktion für Corticosteron $0,8 \%$ und für Cortison und Cortisol weniger als $0,1 \%$.

Zur Bestimmung der Interferenz von Cortisol, Cortison und Corticosteron wurden $\mathbf{5 0}$ ng Corticosteron und Cortison sowie $250 \mathrm{ng}$ Cortisol mit verschiedenen Men-
Tab. 3. Vergleichende Aldosteronbestimmung gleicher Plasmaproben in zwei verschiedenen Laboratorien.

\begin{tabular}{ccc}
\hline $\begin{array}{l}\text { Marburg } \\
\text { [ng/1] }\end{array}$ & $\begin{array}{l}\text { Tibingen } \\
\text { [ng/l] }\end{array}$ & $\begin{array}{c}\text { Differenz } \\
\text { in \% }\end{array}$ \\
\hline 430 & 376 & 12,5 \\
107 & 107 & 0 \\
580 & 350 & 39,7 \\
220 & 170 & 22,8 \\
117 & 158 & 26,0 \\
270 & 294 & 7,0 \\
149 & 169 & 11,8 \\
638 & 606 & 5,0 \\
198 & 173 & 12,8 \\
342 & 488 & 30,0 \\
542 & 457 & 15,6 \\
267 & 266 & 0,1 \\
163 & 217 & 24,8 \\
263 & 240 & 8,8 \\
165 & 152 & 7,8 \\
107 & 55 & 48,6 \\
180 & 134 & 25,5 \\
168 & 212 & 20,7 \\
46 & 64 & 26,3 \\
370 & 275 & 25,5 \\
196 & 197 & 0,5 \\
\hline
\end{tabular}

Mittlere prozentuale Differenz der Einzelwerte

$\pm 17,7 \%$

gen von reinem Aldosteron $(62,5,125,250,500$ und $1000 \mathrm{pg}$ Aldosteron) extrahiert und chromatographiert. Abbildung 3 zeigt, daß eine Trennung des Aldosterons

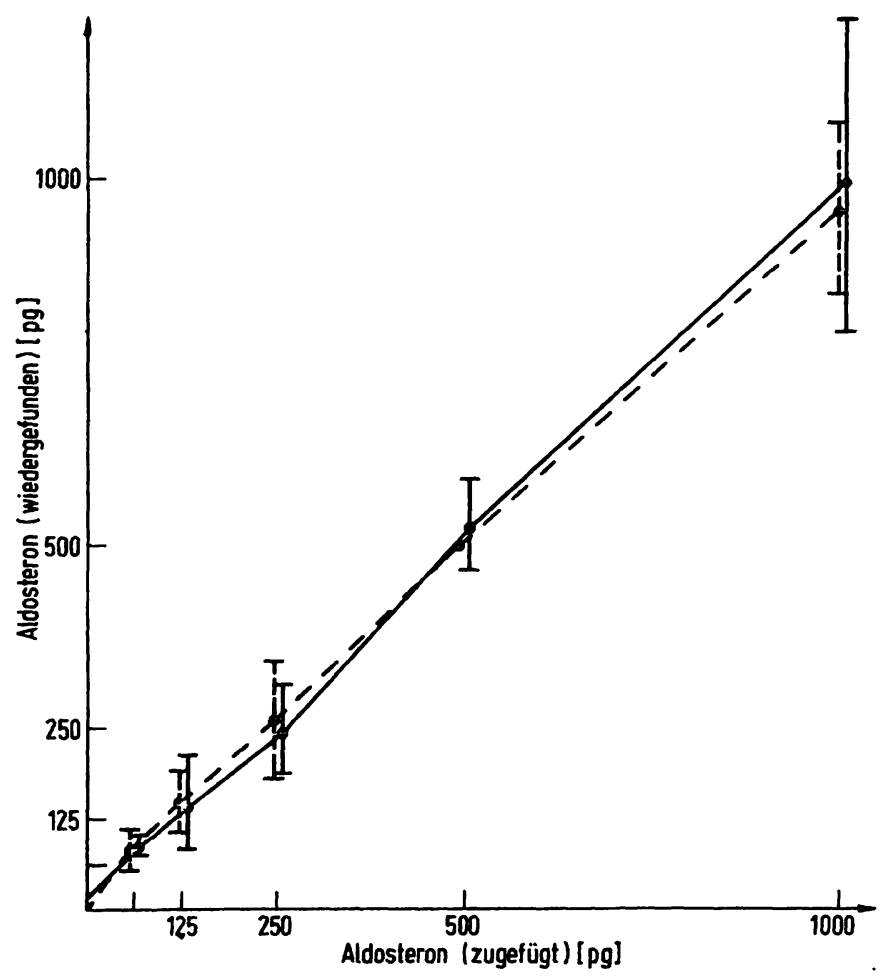

Abb. 3. Wiedergefundenes Aldosteron in pg nach Extraktion und Chromatographie von 62,5-100 pg Aldosteron bei gleichzeitiger Zugabe von 50 ng Cortison, 50 ng Corticosteron und $250 \mathrm{ng}$ Cortisol. (Durchgezogene Linie: Wiedergefundenes Aldosteron in $\mathrm{pg}$ bei alleiniger Zugabe von $\mathrm{Al}$ dosteron; gestrichelte Linie: Wiedergefundenes Aldostoron in pg bei gleichzeitiger Zugabe von Cortison, Corticosteron und Cortisol). Die senkrechten Striche an den einzelnen Punkten entsprechen der Standardabweichung. 


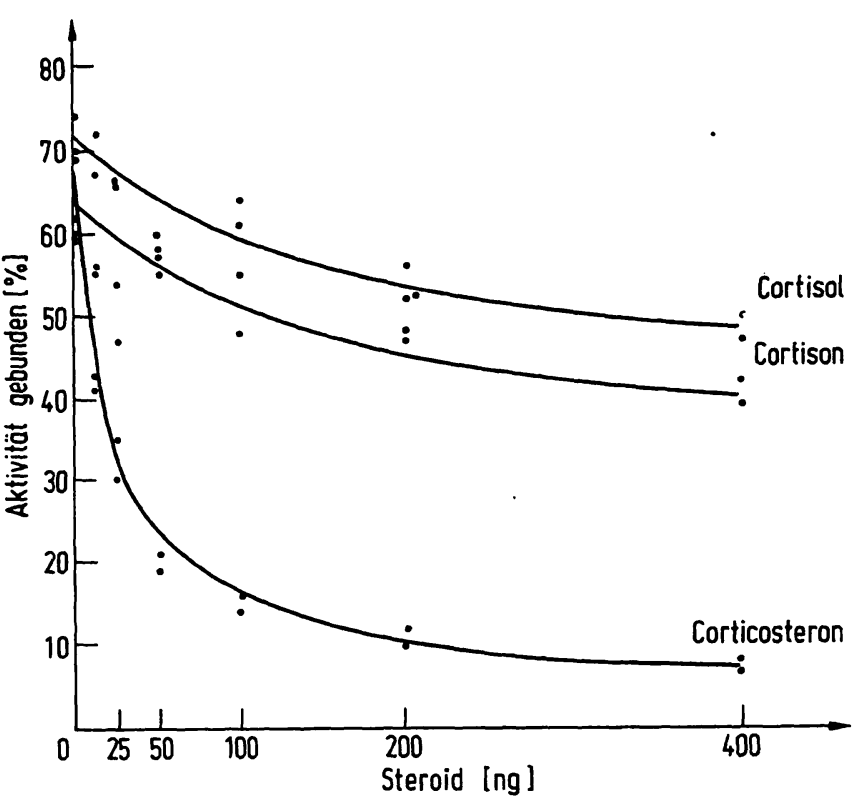

Abb. 4. Kreuzreaktion des Antiserums mit 0-400ng Cortison, Corticosteron und Cortisol.

von den ebenfalls mit dem Antikörper reagierenden Steroiden, die im Plasma in wesentlich höherer Konzentration vorliegen, durch die Chromatographie gewährleistet ist.

\section{Kritische Punkte des Verfahrens}

Bei der Durchführung der Methode ist generell zu beachten, daß immer dann, wenn während der Bestimmung Aldosteron in Lösung vorliegt, die angegebenen Zeiten genau eingehalten werden müssen und alle Proben gleichmäßig behandelt werden. Während der Extraktion des Aldosterons aus dem Plasma ist ein sorgfältiges, gleichmäßiges Schütteln und eine exakte Trennung der beiden Phasen notwendig. Während des radioimimunologischen Ansatzes muß die Inkubationszeit der Proben exakt eingehalten werden. Kritisch ist die Kontaktzeit bei Zugabe der Kohle-Dextran-Suspension bei $4^{\circ} \mathrm{C}$, die genau 10 Minuten betragen muß. Für den immunologischen Ansatz wurden Plastikröhrchen statt Glasgefäße benutzt. Die Impulszählung erfolgte ebenfalls in Plastikgefäßen, die nur einmal verwendet wurden. Das Antiserum darf nicht mehrmals eingefroren und wieder aufgetaut werden. Dagegen kann man das verdünnte Antiserum ebenso wie die Aldosteron-Standardlösungen etwa 1 Woche lang bei $+4^{\circ} \mathrm{C}$ aufbewahren.

\section{Normalwerte}

Bei 35 Gesunden lag die radioimmunologisch bestimmte Plasmaaldosteron-Konzentration zwischen 70 und $250 \mathrm{ng} / 1$. Der Mittelwert betrug $154 \pm 57 \mathrm{ng} / 1$ (Tab. 4). Zwischen männlichen und weiblichen Probanden ergab sich kein signifikanter Unterschied. Die Werte wurden morgens 2-4 Stunden nach dem Aufstehen bei ambulanten Personen (Studenten und Klinikpersonal) ermittelt. Es bestand keine Kostbeschränkung.
Tab. 4. Aldosteronkonzentration im Plasma bei 35 gesunden Probanden (Mittelwert 154,3 $\pm 57,2 \mathrm{ng} / 1$ )

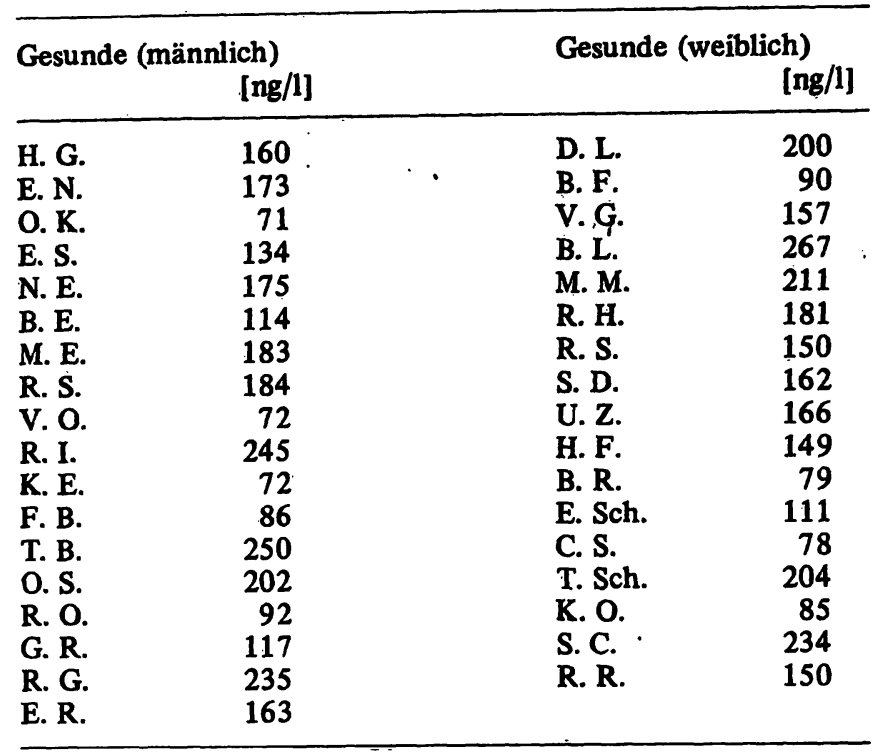

$\bar{x} \pm s=154 \pm 57 \mathrm{ng} / \mathrm{l}$

Aldosteronkonzentration im Plasma bei Morbus Addison und aldosteronsezernierenden Adenomen

Bei 3 Patienten mit unbehandelter Nebennierenrindeninsuffizienz war die radioimmunologisch bestimmte $\mathrm{Al}$ dosteronkonzentration im Plasma auf Werte unter $10 \mathrm{ng} / 1$ vermindert. Zwei Patienten mit operativ und histologisch bestätigten aldosteronsezernierenden $\mathrm{Ne}$ bennierenrindenadenomen wiesen eine Plasmakonzentration von $640 \mathrm{bzw} .920 \mathrm{ng} / \mathrm{l}$ auf (Tab. 5).

Tab. 5. Plasmaaldosteronkonzentration bei Patienten mit Morbus Addison und aldosteronbildenden NebennierenrindenAdenomen.

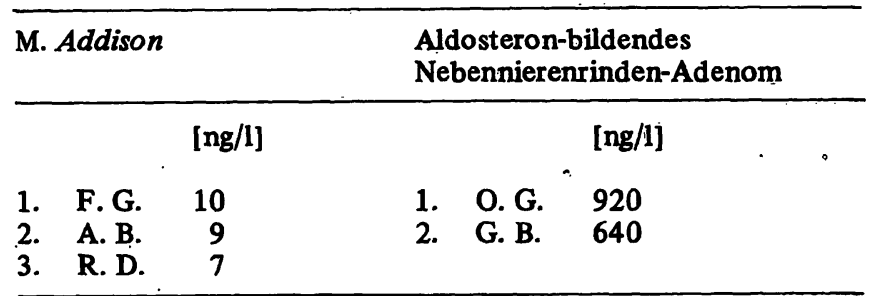

\section{Diskussion}

Das von uns für die radioimmunologische Bestimmung von Aldosteron entwickelte Verfahren kombiniert die Vorteile der von Bayard et al (1) und von Mayes et al (2) angegebenen Methoden. Die Extraktion des Aldosterons aus dem Plasma erfolgte mit Dichlormethan, die Trennung von anderen Steroiden (Cortisol, Corticosteron) durch Papierchromatographie in BenzolMethanol-Wasser. Der dabei eintretende Aldosteronverlust von im Durchschnitt 50\% wurde dadurch genau erfaßt und bei der Berechnung eliminiert, daß jedem Ansatz vor der Chromatographie eine bekannte Menge von markiertem Aldosteron zugesetzt wurde. Für den 
radioimmunologischen Ansatz wurde Tris-Acetatpuffer von $\mathrm{pH}$ 7,4 mit Lysozym und für die Trennung von freiem und gebundenem Aldosteron Kohle-Dextran verwandt.

Die von uns nach der Methode von Bayard et al (1) vom Kaninchen gewonnenen Antikörper gegen Aldosteron zeigen eine ausreichende Spezifität, wiesen aber, wie auch schon von Bayard (1) sowie Mayes (2) gezeigt, eine Kreuzreaktion mit Corticosteron, Cortison und Cortisol auf. Durch die Papierchromatographie konnte Aldosteron gut von diesen störenden Steroiden getrennt werden. Das gewonnenè Antiserum wies in einer Verdünnung von 1:400 eine Bindung von 50-70\% Aldosteron auf.

Die Methode erlaubt den Nachweis von Aldosteron aus einer Plasmamenge von $2 \mathrm{ml}$. Die Empfindlichkeit der Methode gestattet den Nachweis von $50 \mathrm{ng} / 1$ Aldosteron im Plasma. Zur Bestimmung noch niedrigerer Aldo. steron-Konzentrationen ist der Einsatz einer größeren Plasmamenge erforderlich. Die Bestimmung dauert insgesamt 8 Tage. Von einer Med.-techn. Assistentin können pro Tag 8 Bestimmungen durchgeführt werden.

Voraussetzung für eine gute Reproduzierbarkeit der Werte ist die Beachtung einer Reihe von kritischen Punkten bei der Durchführung der Methode, die noch mehr Sorgfalt als andere radioimmunologische Hormonbestimmungen erfordert und besonders die Extraktion des Aldosterons aus dem Plasma betrifft. Bei vergleichenden Untersuchungen über die Reproduzierbarkeit der Werte in 2 Laboratorien mit dem gleichen Antiserum ergab sich, daß die mittlere prozentuale Differenz der Einzelwerte bei 18\% lag.

Bei 35 Gesunden erhielten wir vormittags nach 2-4 stündiger aufrechter Körperhaltung eine mittlere Aldosteronkonzentration im Plasma von $154 \pm$ $57 \mathrm{ng} / \mathrm{l}$. Diese Werte liegen geringfügig höher als die von Mayes et al (2) sowie Bayard et al (1) gefundenen Werte (122 bzw. $132 \mathrm{ng} / \mathrm{l})$. In Ruhelage findet sich unter Normalkost ein niedrigerer Wert, den Mayes (1) und Bayard et al (3) mit 13 bzw. $74 \mathrm{ng} / \mathrm{l}$ bestimmten.

Bei 3 von uns untersuchten Patienten mit Morbus Addison war die Aldosteronkonzentration im Plasma auf Werte unter $10 \mathrm{ng} / \mathrm{l}$ vermindert. Bei 2 Patienten mit Aldosteron-sezernierendem Adenom der Nebennierenrinde fand sich eine auf das 4-6fache erhöhte Aldosteronkonzentration im Plasma.

\section{Danksagung}

Den Med.-techn. Assistentinnen Frl. Peter und Frau Gaco sagen wir für ihre exakte und fleißige Mitarbeit herzlichen Dank.

\section{Literatur}

1. Bayard, F., Beitins, J. Z., Kowarski, A. \& Migeon, C. J. (1970), J. Clin. Endocrinol. Metab. 31, 1-6.

2. Mayes, D., Furuyama, S., Kem, D. C. \& Nugent, C. A. (1970), J. Clin. Endocrinol. Metab. 30, 682-685.

3. Bayard, F., Beitins, J. Z., Kowarski, A. \& Migeon, C. J. (1970), J. Clin. Endocrinol. Metab. 31, 507-510.

4. Goodfriend, T. L., Levine, I. \& Fassman, G. D. (1964) Science 144, 1344-1346.

5. Gocke, D. J., Gerten, J. Sherwood, L. M. \& Laragh, J. H. (1969), Circulat. Res. 24-25, Suppl. 1, 131-146.

6. Hais, J. M. \& Macek, K. (1958), Handbuch der Papierchromatographie 1. Aufl., Jena VEB Gustav Fischer Verlag.

7. Bush, J. E. (1961), The Chromatographie of Steroids Pergamon Press, Oxford.

8. Neher, R. (1959), Chromatographic Reviews (Lederer, M., ed.), vol. 1, p. 99-192, Elsevier Publishing Company, Amsterdam.

9. Rodbard, D., Bridson, W. \& Rayford, P. L. (1969), J. Lab. Clin. Med. 74, 770-781.

10. Rössler, R., Habilitationsschrift 1973, Tübingen.

Dr. Friedrich Klumpp Prof. Dr. D. Klaus $355 \mathrm{Marburg} / \mathrm{L}$. Medizinische Poliklinik Robert Koch Straße ?

Priv. Doz. Dr. R. Rössler 74 Tübingen Medizinische Poliklinik Liebermeisterstraße 14 